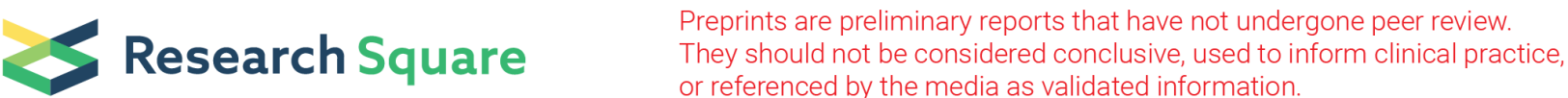

\section{Fine comparision of the efficacy and safety between GB242 and infliximab in patients with active moderate to severe rheumatoid arthritis: a randomized, double- blind, phase III study}

\section{Zhanguo Li ( $\nabla$ zgl21888@163.com )}

Peking University People's Hospital

\section{Yanying Liu}

Peking University People's Hospital

Shengyun Liu

The First Affiliated Hospital of Zhengzhou University

Lin Liu

Xuzhou General Hospital

Xiaowei Gong

The First Hospital of Qiqihar

Ju Liu

Jiujang No 1 Peoples Hospital: Affiliated Jiujiang Hospital of Nanchang University

Lingyun Sun

The Affiliated Hospital of Nanjing University of Medical School

Xiumei Liu

First Hospital of Shanxi Medical University

Lijun Wu

People's Hospital of Xinjiang Uygur Autonomous Region

\section{Linjie Chen}

The First Affiliated Hospital of Bengbu Medical College

\section{Ling Wang}

Zaozhuang Municipal Hospital

\section{Li Luo}

The First Affiliated Hospital of Xinjiang Medical University

Jinying Lin

People's Hospital of Guangxi Zhuang Autonomous Region

\section{Ning Tie}

The Affiliated Hospital of Inner Mongolia University

\section{Zhenyu Jiang}

Jilin University First Hospital

\section{Jian Wu}

The First Affiliated Hospital of Soochow University 


\section{Fuai Lu}

The First Affiliated Hospital of Baotou Medical college

\section{Hongsheng Sun}

Shangdong Province Hospital Affiliated to Shandong First Medical University

\section{Xiaomei Li}

Anhui Provincial Hospital

\section{Niansheng Yang}

The First Affiliated Hospital, Sun Yat-sen University

\section{Kexia Chai}

Qinghai University Affiliated Hospital

\section{Hua Wei}

Northern Jiangsu People's Hospital

\section{Zhanyun Da}

Affiliated Hospital of Nantong University

\section{Cheng Zhao}

First Affiliated Hospital of Guangxi Medical University

\section{Lie Dai}

Sun Yat-Sen University 2nd Affiliated Hospital: Sun Yat-Sen Memorial Hospital

\section{Youlian Wang}

Jiangxi Provincial People's Hospital

\section{Guixiu Shi}

The First Affiliated Hospital of Xiamen University

\section{Zhenchun Zhang}

Linyi People's Hospital

\section{Hui Song}

Beijing Jishuitan Hospital

\section{Yingxue Cathy Liu}

Genor biopharma Co.,Ltd

\section{Qian Guo}

Genor Biopharma Co.,Ltd.

\section{Research article}

Keywords: rheumatoid arthritis, infliximab, biosimilar

Posted Date: May 6th, 2021

DOl: https://doi.org/10.21203/rs.3.rs-436813/v1

License: (c) (1) This work is licensed under a Creative Commons Attribution 4.0 International License. Read Full License 


\section{Abstract}

Background: This double-blind, active-controlled, randomized, multicenter phase III study evaluated the efficacy, safety and immunogenicity of GB242, an infliximab biosimilar, vs infliximab (Remicade ${ }^{\circledR}$ ) reference product in patients with moderate to severe active rheumatoid arthritis (RA) combination with methotrexate (MTX) therapy.

Methods: Patients randomised in a 1:1 ratio to receive either GB242 or INF (3 mg/kg intravenous at weeks 0, 2, 6, 14 and 22). MTX was given as an oral weekly dose of $10-25 \mathrm{mg} /$ week with folic acid of 5-10 mg/week, 30 times totally. The primary end point was the American College of Rheumatology 20\% (ACR20) response rate at week 30. Therapeutic equivalence of clinical response according to ACR20 criteria was declared if the two-sided $95 \% \mathrm{Cl}$ for the treatment difference was within $\pm 14 \%$. Statistical analysis was also performed with a two-sided $95 \% \mathrm{Cl}$ using $\pm 14 \%$ margin. Secondary endpoints included the proportion of patients achieving a week $30 \mathrm{ACR}$ 50 response, ACR70 response, change in Disease Activity Score 28 (DAS28), as well as safety and immunogenicity of GB242.

Results: 570 subjects were randomized into GB242 (N=285) or INF (N=285). Finally, 283 subjects in each group were analysed. The primary endpoint was the ACR20 response. In the full analysis population, $177(62.54 \%)$ of 283 patients in the GB242 group (95\% Cl 56.62\% - 68.20\%) and $161(56.89 \%)$ of 283 patients in the INF group $(95 \% \mathrm{Cl} 50.90 \%-62.74 \%)$ reached ACR20 at week 30 . The difference between the two groups was $5.65 \%$ with a $95 \% \mathrm{Cl}$ of -2.48 to 13.74 , which was within the predefined equivalence margin. The ACR20 response was also similarly shown in the PPS; ACR20 was $71.73 \%$ for GB242 and $66.52 \%$ for INF, respectively. The difference between the two groups was $5.21 \%$ with a $95 \% \mathrm{Cl}$ of -3.33 to 13.6 , which was within the predefined equivalence margin. The second endpoints outcomes such as ACR50 response rate, ACR70 response rate and DAS28 were similar between GB242 and INF. ACR50 response for the FAS was $37.12 \%$ for GB242 and $32.86 \%$ for INFखACR50 response for the PPS was $42.62 \%$ for GB242 and $38.63 \%$ for INF. ACR70 response for the FAS was $19.79 \%$ for GB242 and $16.96 \%$ for INF, ACR70 response for the PPS was $22.78 \%$ for GB242 and $20.63 \%$ for INF, respectively. The incidence of treatment-emergent adverse events was comparable ( $77.4 \%$ in GB242 vs $80.2 \%$ in INF) and detection of antidrug antibodies (ADA) to infliximab up to week 30 (60.8\% in GB242 vs $59.4 \%$ in INF) was comparable.

Conclusions: The efficacy of GB242 appeared to be slightly better than that of INF in terms of ACR20, ACR50, ACR70 at week 30, but the difference was not statistically significant. Moreover, GB242 was well tolerated, with a similar immunogenicity and safety profile comparable to INF.

Trial registration: CTR20170127, registered 28 July 2017; NCT04178850, registered 26 Nov 2017-Retrospectively registered, https://clinicaltrials.gov/ct2/show/NCT04178850?term=GB242\&draw=2\&rank=1

\section{Research In Context}

\section{Evidence before this study}

Preclinical data and phase I study of GB242 support that GB242 could be an effective therapy in patients with moderate to severe active rheumatoid arthritis. Globally, several clinical studies are ongoing to find a more economical therapy of rheumatoid arthritis than infliximab. 
GB242 phase III clinical study evaluate the efficacy and safety of GB242, an infliximab biosimilar, vs infliximab (Remicade ${ }^{B}$ ) reference product in patients with moderate to severe active rheumatoid arthritis (RA). The data show that GB242 demonstrate equivalent efficacy to INF at week 30, with a similar immunogenicity. Moreover, GB242 is well tolerated, with a safety profile comparable to infliximab.

\section{Implications of all the available evidence}

Our findings suggest GB242 is clinically active and well tolerated in patients with moderate to severe active RA. GB242 is a low-cost biosimilar drug with the potential to have a large impact on RA outcomes globally.

\section{Background}

Rheumatoid arthritis (RA) is a chronic inflammatory autoimmune disease characterized by high morbidity and disabilities leading to a significant cost to both individual and the society.[1, 2] Methotrexate (MTX) remains the anchor drug in RA according to ACR-EULAR 2019. However, only half of the patients respond adequately to MTX. $[3,4]$ While tumour necrosis factor-a (TNFa) inhibitors such as infliximab have showed clinical benefit in patients with RA, the high economic burden due to hospitalisation and long term therapy is considerable.[5, 6]

A biosimilar is a biologic drug that contains a high similarity of the structure and function to a reference or original biological medicinal product. $[7,8,9]$ The definition of a biosimilar include three aspects: Firstly, the similarity of molecular level in terms of a comprehensive physicochemical and biological characterisation was demonstrated. [10] Secondly, bioequivalence of pharmacokinetic (PK) need to be proved [11], and finally, the safety and efficacy of clinical equivalence are done to compare with the reference product. [12]

GB242 is an immunoglobulin (Ig) G1 chimeric human-murine monoclonal antibody biosimilar to infliximab reference product (INF). GB242 is produced in the same type of cell-line and has an identical amino acid sequence to INF. The phase I study had shown that GB242 and the INF were similar on the molecular level and bioequivalent in the healthy adult volunteers in China. [13]

Based on the data described, this study now reports a phase III study to assess efficacy equivalence and overall safety of GB242 versus INF in active moderate to severe RA patients.

\section{Methods}

\section{Patients}

Patients who were 18-75 years old with RA according to the 2010 American College of Rheumatology (ACR) classification criteria were recruited. Patients should fulfil the following criteria, patients had to have $\geq 4$ swollen and $\geq 6$ tender joints, and with erythrocyte sedimentation rate (ESR) $>28 \mathrm{~mm} / \mathrm{h}$ or serum $\mathrm{C}$ reactive protein (CRP) concentration $>1.0 \mathrm{mg} / \mathrm{dl}$. Patients had to have been receiving methotrexate (MTX) therapy for $\geq 3$ months (stable dose of 10-15 mg/week for $\geq 4$ weeks prior to screening). Patients have discontinued other diseasemodifying antirheumatic drugs (DMARDs) for at least 4 weeks except for MTX prior to the first dose of study drug. Use of leflumide also required a washout period prior to the first dose of study drug. Patients were 
permitted to receive oral glucocorticoids (equivalent to $\leq 10 \mathrm{mg}$ daily prednisolone) for $\geq 4$ weeks prior to screening. More detailed eligibility criteria are available in the supplementary material.

\section{Study design}

This study is a phase III, randomised, double-blind, multicentre parallel group study. The study (ClinicalTrials.gov NCT04178850) was conducted according to the Declaration of Helsinki and the International Committee on Harmonisation good clinical practice and all applicable regulatory requirements. The study protocol was reviewed and approved by independent ethics committees of each study site. All patients provided written informed consent.

The study was conducted at 29 centres in China. Patients were randomized 1:1 to receive $2 \mathrm{~h}$ intravenous infusion of either $3 \mathrm{mg} / \mathrm{kg}$ of GB242 (Yuxi Genor Biotechnology Co., Ltd.) or INF囚Cliag AG囚 at weeks 0, 2, 6, 14, 22 , in total 5 times. MTX was given as an oral weekly dose of $10-25 \mathrm{mg} /$ week with folic acid of $5-10 \mathrm{mg} /$ week, 30 times totally. Non-steroidal anti-inflammatory drugs and corticosteroids ( $\leq 10 \mathrm{mg}$ prednisolone) were allowed if taken for a stable dose for 4 weeks before randomisation. Other diseases modifying anti-rheumatic drugs except for MTX were prohibited during the study. Other therapeutic biologic agents should be banned 3 months before and throughout the trial.

\section{Endpoints}

The primary endpoint was the percentage of patients achieving ACR criteria for $\geq 20 \%$ clinical improvement (ACR20) at week30 in the per-protocol set (PPS) and the full analysis set (FAS), as determined by ACR20 response criteria. Equivalence of efficacy was concluded if the $95 \% \mathrm{Cls}$ for treatment difference were within $\pm 14 \%$ at week 30 .

Second endpoints at weeks 2, 4, 6, 12, 14 and 22 included ACR20 (other than week 30), ACR50 ( $\geq 50 \%$ clinical improvement), and ACR70 ( $\geq 70 \%$ clinical improvement) response rates; the changes from baseline in DAS28 (CRP) at weeks 14 and 30. Patients were considered to be in DAS remission when DAS28-CRP was < 2.6, and ACR remission was defined based on scores for the tender joint count, swollen joint count, CRP, ESR and HAQ-DI. Additional secondary endpoints included immunogenicity and safety.

Immunogenicity tests were evaluated by serum antidrug antibodies (ADAs) and neutralising antibodies (NAbs) to infliximab and GB242 at weeks 2, 6, 14, 22 before infusion and weeks 30, respectively. ADAs were measured by a bridging electrochemiluminescent (Bridging-ECL) immunoassay utilizing the Meso Scale Discovery platform (MSD, Shanghai, China). Those who were ADA-positive were additionally assessed for neutralising antibodies.

Safety endpoints included incidence and type of adverse events (AEs) and serious AEs, clinical laboratory abnormalities, including incidence, severity, type, infusion-related reactions. Other safety assessments included vital signs and abornamalities of other physical examinations. AEs were coded by the Medical Dictionary for Regulatory Activities, version 22.0 and severity was classified as 1- 5 according to Common Terminology Criteria for Adverse Events (CTCAE) version 4.03. Treatment-emergent AEs (TEAEs) were assessed throughout the study and defined as any $A E$ that occurred, or any pre-existing $A E$ that worsened, after the beginning of study treatment. Latent or active tuberculosis (TB) were screened by an interferon $y$-release assay using QuantiFERONTB Gold in tube (QTF-TB Gold-IT, Genor, China) and chest x-ray at screening, weeks 14 and 30. 


\section{Statistical analysis}

All primary efficacy analyses were performed on both the PPS and the FAS. Sample size was calculated according to the following criterion: the randomized equivalence trial to INF is based on the expected response rate of $60 \%$.[14] Sample size was calculated by specifying a two-sided a level of 0.05 , power of $80 \%$ and a twosided equivalence margin of $15 \%$, a sample size required 516 patients in the per-protocol (PP) population final analysis. With a $10 \%$ dropout rate, a sample size of at least 568 randomised patients were required for the PPS. The equivalence margin was determined using data from several INF studies [15-16] and regulatory guidelines [17-18].

All efficacy outcome was analysed using the PPS and FAS [19]. FAS included all randomised patients who received at least one dose of GB242 or INF. The primary efficacy was to measure equivalence of ACR20 of GB242 and INF at week 30. Analysis of ACR50 and ACR70 was also done in PPS and FAS; DAS28 was performed in the PPS and FAS and DAS28 was analysed by using MMRM to calculate the mean value and the corresponding $95 \%$ confidence interval.

Safety analysis was done in the number of patients who had a particular AE in the safety analysis set (SS; those who received at least one dose of GB242 or INF). ADA analysis was also done in those patients having incident ADA up to weeks 30 from the SS.

\section{Results}

\section{Patients}

The first patient was screened in October 2017; the last week 30 evaluation was performed in March 2020. Baseline demographics and disease status were comparable between GB242 and INF (table 1). Of the 570 randomized patients, 491 completed the 30-week study period and, of these, 65 patients were excluded from the PP population due to major protocol violations. Discontinuation in randomized patients was primarily due to AEs (8.9\%) and patient withdrawal of consent (4.1\%) (Figure 1).

\section{Efficacy}

The ACR20 response over time is shown in figure 2. The ACR20 response at each visit was similar between GB242 and INF; the two time-response curves were determined to be equivalent. Moreover, the primary endpoint of ACR20 response at week 30 was equivalent between GB242 and INF group. The ACR20 response for the FAS was $62.54 \%$ for GB242 and $56.89 \%$ for INF, respectively. The $95 \% \mathrm{Cl}$ for the rate difference was $-2.48 \%$ to $13.74 \%$, which was within the prespecified equivalence margin of $\pm 14 \%$. The ACR20 response was also similarly shown in the PPS; ACR20 was $71.73 \%$ for GB242 and $66.52 \%$ for INF, respectively. Thus, the equivalence of GB242 compared with INF was concluded. Other efficacy outcomes such as ACR50 or ACR70 were also similar in the FAS and PPS (figure 3). ACR50 response for the FAS was $37.12 \%$ for GB242 and $32.86 \%$ for INF『ACR50 response for the PPS was $42.62 \%$ for GB242 and $38.63 \%$ for INF. ACR70 response for the FAS was $19.79 \%$ for GB242 and $16.96 \%$ for INF, ACR70 response for the PPS was $22.78 \%$ for GB242 and $20.63 \%$ for INF, respectively.

The data shows that from baseline to week 30 , the changes of each efficacy component used for calculating ACR responses or DAS28 activity were similar between GB242 and INF (supplementary appendix S1). The overall 
ACR20 response rate was lower in the ADA-positive subgroup compared with the ADA-negative subgroup, but was also similar between GB242 and INF within each ADA subgroup (58.67\% vs $54.26 \%$, ADA- positive subgroup; $74.68 \%$ vs $65.88 \%$, ADA- negative subgroup \supple- mentary appendix S2), and there was not significant $(P=0.7408)$ between the ADA- positive subgroup and ADA- negative subgroup .

The response for DAS28 over time, the proportion of remission, low disease activity (LDA), moderate and high disease activity by DAS28 is shown in figure 4. The change of DAS28 (CRP) and DAS28 (ESR) over time was similar between GB242 and INF (Figure 4A), and the proportion of remission, LDA, moderate and high disease activity by DAS28 (CRP) at weeks 30 was $43 \%$ for GB242 and $42.5 \%$ for INF, $19.8 \%$ for GB242 and $18 \%$ for INF, $34.6 \%$ for GB242 and 36.9\% for INF, 2.5\% for GB242 and 2.6\% for INF, respectively (Figure 4B). Overall, the efficacy of GB242 appeared to be slightly better than that of INF, but the difference was not statistically significant.

\section{Immunogenicity}

The incidence of ADA was similar between GB242 and INF at all measured time points (supplementary appendix

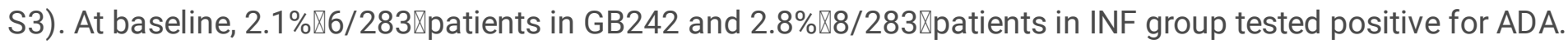
Patients who developed ADA up to week 6 were $7.4 \%$ (21/ 283) in the GB242 treatment group and $11.0 \%$ $(31 / 283)$ in the INF treatment group and 60.8\% (172/ 283) in the GB242 treatment group and 59.4\% (168/283) in the INF treatment group, respectively, at week 30. Patients also were tested NAb-positive. The incidence of NAb was similar between GB242 and INF treatment group at all measured time points (supplementary appendix S3). There was a similar trend between two treatment groups. NAb positive rate at baseline was $0.7 \%$ in the GB242 treatment group and 0 in the INF treatment group, at week 30, 25.4\% (72/283) in the GB242 treatment group and $23 \%(65 / 283)$ in the INF treatment group, respectively.

\section{Safety}

In the safety analysis set, overall treatment-emergent AEs (TEAEs) occurred in 219 (77.4\%) patients in the GB242 treatment group and 227 (80.2\%) patients in the INF treatment group (table 2). The most common TEAEs that occurred were upper respiratory tract infection, increase of white blood cell count and Urinary tract infection. The majority of TEAEs was mild to moderate in severity. The proportion of TEAEs reported in the two groups was similar (GB242 54.1\% vs INF 54.4\%).

All patients with latent TB were undergone prophylactic TB medication, and only one patient with latent TB developed active TB, who was in the INF group.

Infusion-related reactions occurred in 27 (9.5\%) and 23 (8.1\%) patients for GB242 and INF, respectively. There were 1 death reported in GB242 (dead of Traumatic brain injury) and one case of suicide from INF. Overall, the safety profile was comparable between GB242 and INF.

Predictors of treatment response

Patients who had DAS28 $(\mathrm{CRP})<2.6$ in weeks 30 were associated with a rapid increase and then stabilization of CD19+ peripheral B cells in GB242 group as well as in the INF group (Figure 5A). Differently, CD19+ peripheral B cell percentage rose slowly and then remained stable in GB242 group and increased firstly and then fell down in the INF group in patients with DAS28(CRP) $\geq 2.6$ in week 30 (Figure 5B). In both the two groups, CD19+ B cell in 
the DAS28 $($ CRP $)<2.6$ group showed a greater increase at week 30 than that at baseline $(p<0.05)$. Meanwhile, mean immunoglobulin (IgG and IgA) levels showed significant decrease between week 30 and baseline in DAS28<2.6 group $(p<0.05)$.

\section{Discussion}

In this randomised, double-blind study, the equivalence of efficacy, safety and immunogenicity profiles between GB242 and INF was demonstrated.

The primary endpoint was ACR20 response at week 30, where GB242 was shown to be slightly higher than that of INF in the FAS and PPS, the 95\% Cls for treatment difference were within the predefined margins for equivalence of $\pm 14 \%$. Moreover, the efficacy endpoints at all visits were compared, and this study has demonstrated the equivalence of ACR20 response over time. To ensure a credible comparison between GB242 and INF data, we also observe other efficacy outcomes besides the ACR 20 response such as ACR50, ACR70 responses.

all of them showed GB242 seems has slightly higher than INF, but the difference was not statistically significant. Moreover, DAS28 was similar between GB242 and INF, further supporting the biosimilarity of GB242 to INF. Efficacy criteria was also measured according to ADA positive rate and NAb positive rate at week 30 , and no statistically significant differences in responses between GB242 and INF groups were found.

The objective with regard to safety was to demonstrate a comparable safety profile of GB242 and INF. Overall, the incidence of AEs associated with treatments such as upper respiratory tract infection, increasement of white blood cell counts, and latent TB were comparable between GB242 and INF. It is notable that the incidence of active TB was very low (only one patient in INF group), which might be attributed to universal TB prophylaxis for patients with latent TB at screening. The rate of infusion reactions in both treatment groups (4.6\% vs $6.4 \%)$ was comparable. The safety results were similar to those previous studies.

In terms of Immunogenicity, GB242 has demonstrated a comparable profile at all measured time points to that of INF. The incidence of ADA ( $60 \%)$ is similar with recent INF studies. [20-23].

In terms of predictors of treatment response, Increase of CD19 + B cell was regarded as a reliable clinical response biomarkers of RA patients [24]. In patients who achieved disease remission at 30 weeks, the increase in CD19 from baseline at 30 weeks was statistically significant $(p<0.05)$, while in patients who did not achieve disease remission at 30 weeks, the increase in CD19 from baseline at 30 weeks was not statistically significant $(p=0.273)$. This suggests that the increase CD19 expression was predictive of a faster control of pathological activity in patients treated with GB242 and INF, especially in patients who achieved DAS28<2.6 at weeks 30 .

\section{Conclusion}

In conclusion, the efficacy of GB242 appeared to be slightly better than that of INF in terms of ACR20, ACR50, ACR70 at week 30 in active moderate to severe RA patients, but the difference was not statistically significant. Other efficacy end points also show consistently similar results when compared with the originator product. Moreover, GB242 was well tolerated and the safety, immunogenicity profiles were comparable with those of INF. 


\section{Abbreviations}

ACR: American College of Rheumatology

ADA: Antidrug antibodies

AE: Adverse event

Cl: Confidence interval

CRP: C-reactive protein

CTCAE: Common Terminology Criteria for Adverse Events

DAS28-CRP: 28-Joint Disease Activity Score based on C-reactive protein

DAS28-ESR: 28-Joint Disease Activity Score based on erythrocyte sedimentation rate

DMARD: Disease-modifying antirheumatic drug

ESR: Erythrocyte sedimentation rate

EULAR: European League Against Rheumatism

HAQ-DI: Health Assessment Questionnaire-Disability Index

INF: Infliximab

IV: Intravenous

LDA: Low disease activity

MTX: Methotrexate

Nabs: Neutralising antibodies

PK: Pharmacokinetic(s)

Q\#W: Every \# weeks

RA: rheumatoid arthritis

RCT: Randomised controlled trial

SD: Standard deviation

SAE: Serious adverse event

TB: tuberculosis

TEAE: Treatment-emergent adverse event 
TNFa: Tumour necrosis factor-a

\section{Declarations}

All authors declared no conflicts of interest.

\section{Ethics approval and consent to participate}

This study complied with the provisions of the Declaration of Helsinki and the International Conference on Harmonization Good Clinical Practice Guidelines, and it was reviewed and approved by an institutional review board or independent ethics committee(s) at each of the participating investigational sites. Informed consent was provided to all patients prior to undergoing any screening procedures.

\section{Consent for publication}

Not applicable.

\section{Availability of supporting data}

Not applicable.

\section{Competing interests}

The authors declare that they have no competing interests.

\section{Funding}

This study was funded by Genor Biopharma Co., Ltd., China.

\section{Authors' contributions}

Zhanguo Li and Yanying Liu contributed to study conception and design. Yanying Liu and Zhanguo Li contributed to drafting the manuscript and final approval of the version to be published. All coauthors contributed significantly to data collection.

\section{Acknowledgments}

The authors thank the patients and their family who were involved in this study, and the sponsor Genor Biopharma Co., Ltd., China.

\section{Availability of data and materials}

The datasets used and analysed during the current study are available from the corresponding author on reasonable request.

\section{References}

1. Birnbaum H, Pike C, Kaufman R, et al. Societal cost of rheumatoid arthritis patients in the US. Curr Med Res Opin 2010;26:77-90. 
2. Cross $M$, Smith $E$, Hoy $D$, et al. The global burden of rheumatoid arthritis: estimates from the global burden of disease 2010 study. Ann Rheum Dis 2014;73:1316-22.

3. Smolen J S, Landewé R, Bijlsma J W J, et al. EULAR recommendations for the management of rheumatoid arthritis with synthetic and biological disease-modifying antirheumatic drugs: 2019 update[J]. Annals of the rheumatic diseases, 2020.

4. Maini R, St Clair EW, Breedveld F, et al. In fliximab (chimeric anti-tumour necrosis factor alpha monoclonal antibody) versus placebo in rheumatoid arthritis patients receiving concomitant methotrexate: a randomised phase III trial. ATTRACT Study Group. Lancet 1999;354:1932-9.

5. Dörner T, Strand V, Cornes P, et al. The changing landscape of biosimilars in rheumatology. Ann Rheum Dis. 2016;75(6):974-982.

6. Modena V, Bianchi G, Roccatello D. Cost-effectiveness of biologic treatment for rheumatoid arthritis in clinical practice: an achievable target? Autoimmun Rev 2013;12:835-8.

7. Committee for Medicinal Products for Human Use (CHMP), European Medicines Agency (EMA). Guideline on similar biological medicinal products. London: EMA; 2014.

8. US Food and Drug Administration (FDA). Scientific considerations in demonstrating biosimilarity to a reference product. Guidance for industry. Silver Spring, MD: FDA; 2015.

9. Gulácsi, L., Brodszky, V., Baji, P., Kim, H., Kim, S. Y., Cho, Y. Y., \& Péntek, M. (2015). Biosimilars for the management of rheumatoid arthritis: economic considerations. Expert review of clinical immunology, 11(sup1), 43-52.

10. Jung SK, Lee KH, Jeon JW, et al. Physicochemical characterization of Remsima. MAbs 2014;6:1163-77.

11. Park W, Hrycaj P, Jeka S, et al. A randomised, double-blind, multicentre, parallel-group, prospective study comparing the pharmacokinetics, safety, and efficacy of CT-P13 and innovator infliximab in patients with ankylosing spondylitis: the PLANETAS study. Ann Rheum Dis 2013;72:1605-12.

12. Yoo DH, Hrycaj P, Miranda P, et al. A randomised, double-blind, parallel-group study to demonstrate equivalence in efficacy and safety of CT-P13 compared with innovator infliximab when coadministered with methotrexate in patients with active rheumatoid arthritis: the PLANETRA study. Ann Rheum Dis 2013;72:1613-20

13. Zhang T, Chen G, Liu C, et al. A Phase I Study Comparing the Pharmacokinetics, Safety, and Immunogenicity of Proposed Biosimilar GB242 and Reference Infliximab in Healthy Subjects[J]. BioDrugs, 2019, 33(1): 93100.

14. Maini R, St Clair EW, Breedveld F, et al. In fliximab (chimeric anti-tumour necrosis factor alpha monoclonal antibody) versus placebo in rheumatoid arthritis patients receiving concomitant methotrexate: a randomised phase III trial. ATTRACT Study Group. Lancet 1999;354:1932-9.

15. Abe, T., Takeuchi, T., Miyasaka, N., Hashimoto, H., Kondo, H., Ichikawa, Y., \& Nagaya, I. (2006). A multicenter, double-blind, randomized, placebo controlled trial of infliximab combined with low dose methotrexate in Japanese patients with rheumatoid arthritis. The Journal of rheumatology, 33(1), 37-44.

16. Westhovens R, Yocum D, Han J, et al. The safety of infliximab, combined with background treatments, among patients with rheumatoid arthritis and various comorbidities: a large, randomized, placebo-controlled trial. Arthritis Rheum, 2006;54:1075-86 
17. EMA/CHMP. Guideline on the choice of the non-inferiority margin. 2005.

http://www.ema.europa.eu/docs/en_GB/document_library/Scientific_guideline/2009/09/WC500003636.pdf (accessed 6 Mar 2015).

18. US FDA. Guidance for Industry: non-inferiority clinical trials. 2010. http://www.fda.gov/downloads/Drugs/Guidances/UCM202140.pdf (accessed 6 Mar 2015)

19. Statistical Principles for Clinical Trials E9. 1998. http://www.ich.org/fileadmin/Public_Web_Site/IC

20. Yoo D H, Hrycaj P, Miranda P, et al. A randomised, double-blind, parallel-group study to demonstrate equivalence in efficacy and safety of CT-P13 compared with innovator infliximab when coadministered with methotrexate in patients with active rheumatoid arthritis: the PLANETRA study[J]. Annals of the rheumatic diseases, 2013, 72(10): 1613-1620.

21. Bendtzen K, Geborek P, Svenson M, et al. Individualized monitoring of drug bioavailability and immunogenicity in rheumatoid arthritis patients treated with the tumor necrosis factor alpha inhibitor infliximab. Arthritis Rheum 2006;54:3782-9.

22. Pascual-Salcedo D, Plasencia C, Ramiro S, et al. Influence of immunogenicity on the efficacy of long-term treatment with infliximab in rheumatoid arthritis. Rheumatology (Oxford) 2011;50:1445-52.

23. Cohen, S. B., Alten, R., Kameda, H., Hala, T., Radominski, S. C., Rehman, M. I., ... \& Sewell, K. L. (2018). A randomized controlled trial comparing PF-06438179/GP1111 (an infliximab biosimilar) and infliximab reference product for treatment of moderate to severe active rheumatoid arthritis despite methotrexate therapy. Arthritis research \& therapy, 20(1), 1-13.

24. Zecevic L, Mekic M, Subasic D, et al. Expression of NK (CD16+ 56+) and B cells (CD19) Receptor Molecules as a Reliable Clinical Response Biomarkers of SLE and RA Patients Under the Rituximab Treatment[J]. Medical Archives, 2019, 73(6): 374.

\section{Tables}

Table 1. Baseline characteristics the study population. 


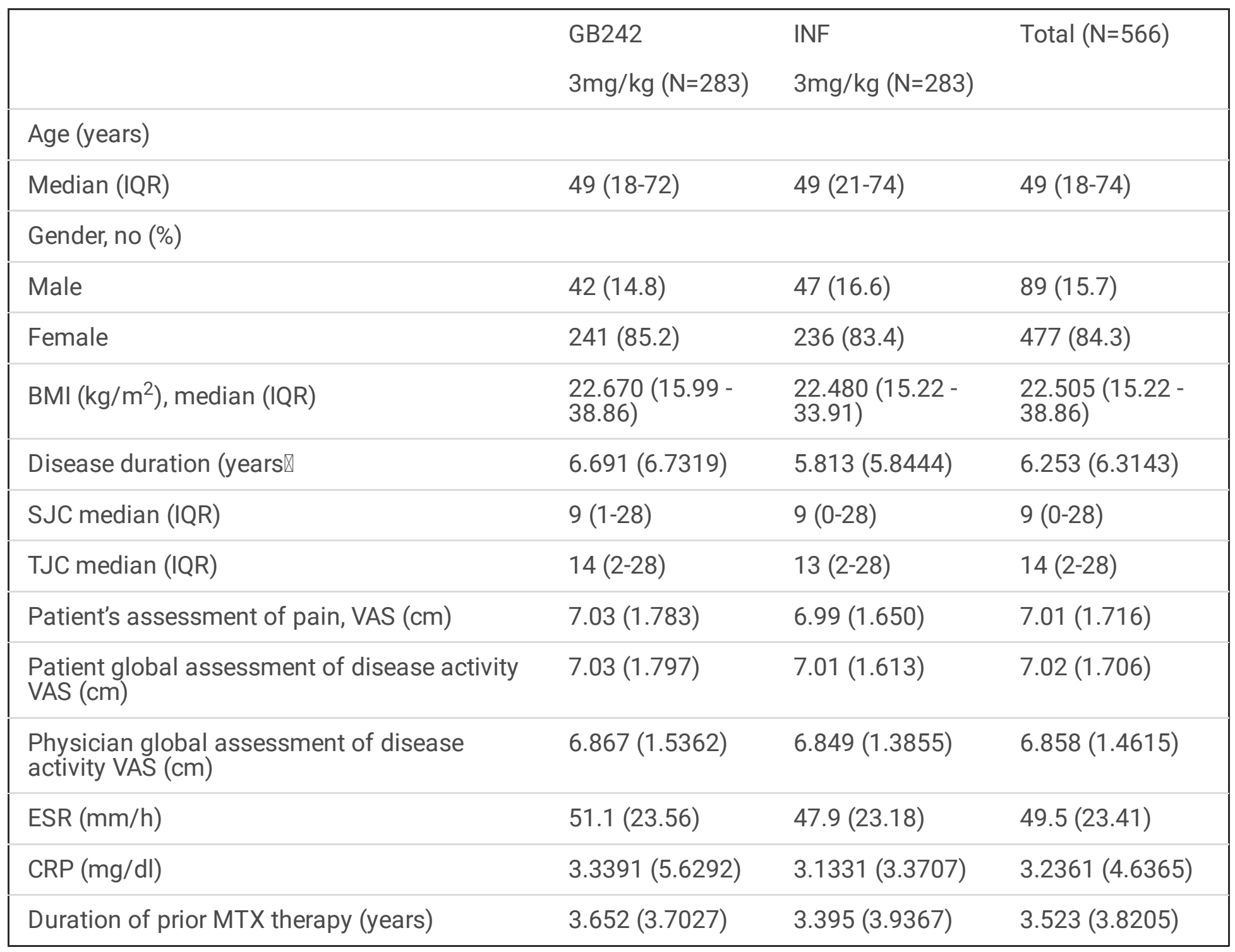

*Except where indicated otherwise, values are the mean (SD).

$\mathrm{BMI}$, body mass index; CRP, C reactive protein; ESR, erythrocyte sedimentation rate; INF, innovator infliximab; MTX, methotrexate; SJC, swollen joint count; TJC, tender joint count.

Table 2 Treatment-emergent adverse events (TEAEs) that were reported in at least 1\% of patients in GB242 and INF group, no (\%). 


\begin{tabular}{|lccc|}
\hline TEAEs & GB242 & INF & Total \\
& $(\mathrm{N}=283)$ & $(\mathrm{N}=283)$ & $(\mathrm{N}=566)$ \\
& $\mathrm{n}(\%)$ & $\mathrm{n}(\%)$ & $\mathrm{n}(\%)$ \\
\hline Any TEAEs, patients (\%) & $219(77.4)$ & $227(80.2)$ & $446(78.8)$ \\
\hline Upper respiratory tract infection & $60(21.2)$ & $68(24.0)$ & $128(22.6)$ \\
\hline White blood cell count increased & $20(7.1)$ & $30(10.6)$ & $50(8.8)$ \\
\hline Urinary tract infection & $18(6.4)$ & $23(8.1)$ & $41(7.2)$ \\
\hline Anemia & $16(5.7)$ & $14(4.9)$ & $30(5.3)$ \\
\hline Hyperlipemia & $14(4.9)$ & $4(1.4)$ & $18(3.2)$ \\
\hline Abnormal liver function test & $14(4.9)$ & $19(6.7)$ & $33(5.8)$ \\
\hline Weight increase & $11(3.9)$ & $8(2.8)$ & $19(3.4)$ \\
\hline Aspartate aminotransferase increased & $11(3.9)$ & $11(3.9)$ & $22(3.9)$ \\
\hline Pyrexia & $11(3.9)$ & $10(3.5)$ & $21(3.7)$ \\
\hline Alanine aminotransferase increased & $10(3.5)$ & $13(4.6)$ & $23(4.1)$ \\
\hline Toothache & $9(3.2)$ & $1(0.4)$ & $10(1.8)$ \\
\hline Cough & $9(3.2)$ & $16(5.7)$ & $25(4.4)$ \\
\hline Hyperuricemia & $7(2.5)$ & $5(1.8)$ & $12(2.1)$ \\
\hline Drug-induced hypersensitivity reaction & $7(2.5)$ & $1(0.4)$ & $8(1.4)$ \\
\hline Neutrophil count decreased & $6(2.1)$ & $7(2.5)$ & $13(2.3)$ \\
\hline Pneumonia & $6(2.1)$ & $5(1.8)$ & $11(1.9)$ \\
\hline Rash & $6(2.1)$ & $4(1.4)$ & $10(1.8)$ \\
\hline Dizziness & $6(2.1)$ & $5(1.8)$ & $11(1.9)$ \\
\hline Rheumatoid arthritis & $4(1.4)$ & $7(2.5)$ & $11(1.9)$ \\
\hline Hypertension & $4(1.4)$ & $6(2.1)$ & $10(1.8)$ \\
\hline Lymphocyte count decreased & $3(1.1)$ & $6(2.1)$ & $9(1.6)$ \\
\hline Nausea & $3(1.1)$ & $7(2.5)$ & $10(1.8)$ \\
\hline Allergic dermatitis & $2(0.7)$ & $7(2.5)$ & $9(1.6)$ \\
\hline Infusion related reactions & $6(2.1)$ & $8(1.4)$ \\
\hline
\end{tabular}

The total number of treatment-emergent adverse events count included all related patient events. 
*In GB242 group for safety analyses included patients who received at least one (full or partial) dose of GB242, irrespective of their randomisation. INF, infliximab; RA, rheumatoid arthritis.

\section{Figures}

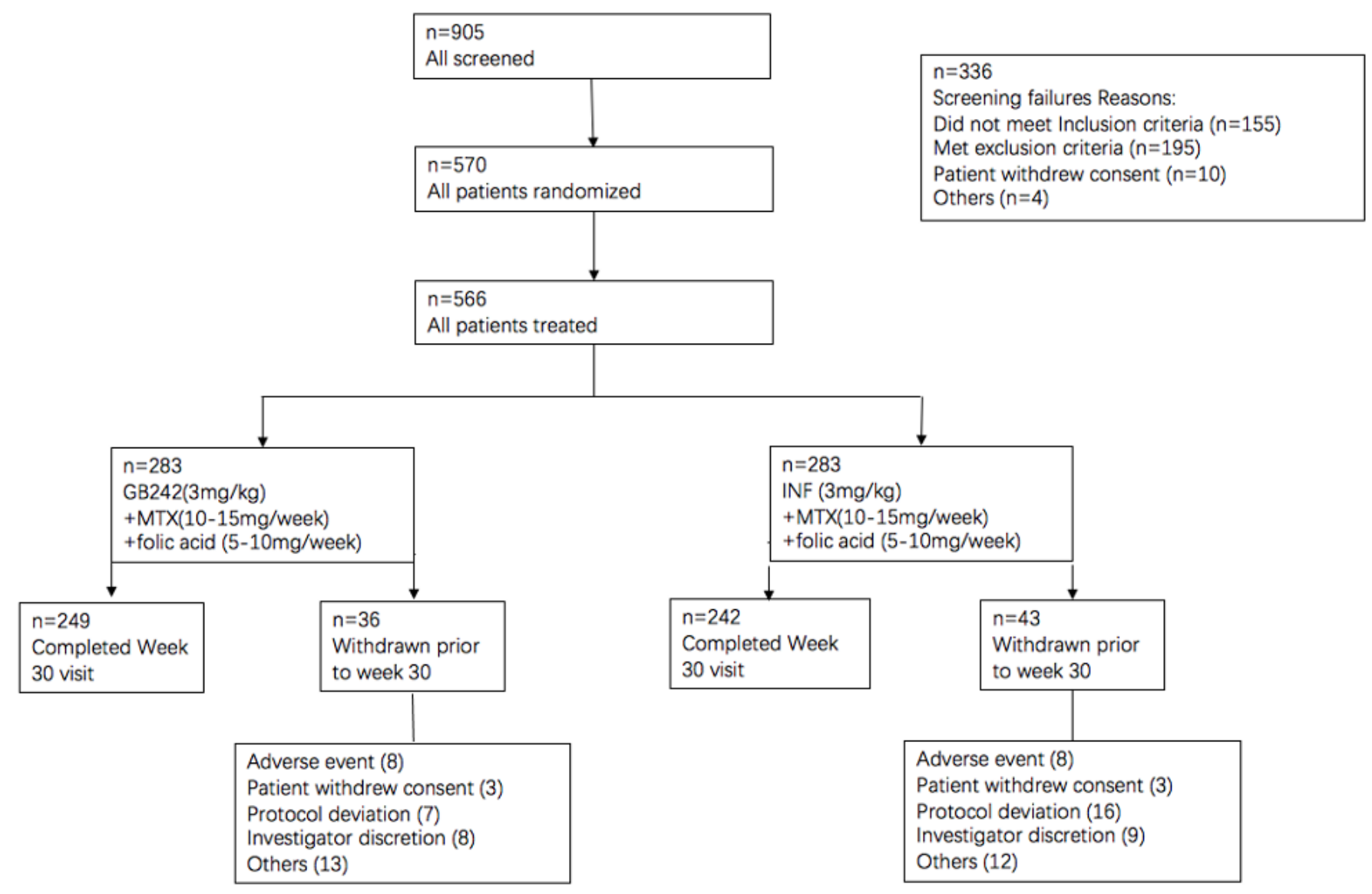

\section{Figure 1}

Disposition flow chart of the study population. A total of 905 patients were screened for the study, and 566 eligible patients were randomised into a GB242 group $(n=283)$ or an infliximab reference product (INF) group $(n=283)$ to receive $3 \mathrm{mg} / \mathrm{kg}$ of GB242 or INX, respectively, coadministered with methotrexate (MTX) and folic acid. The full analysis set (FAS) for GB242 is $n=283$ and infliximab reference product (INF) $n=283$. The perprotocol set (PPS) for GB242 is $n=237$ and INF $n=233$. 


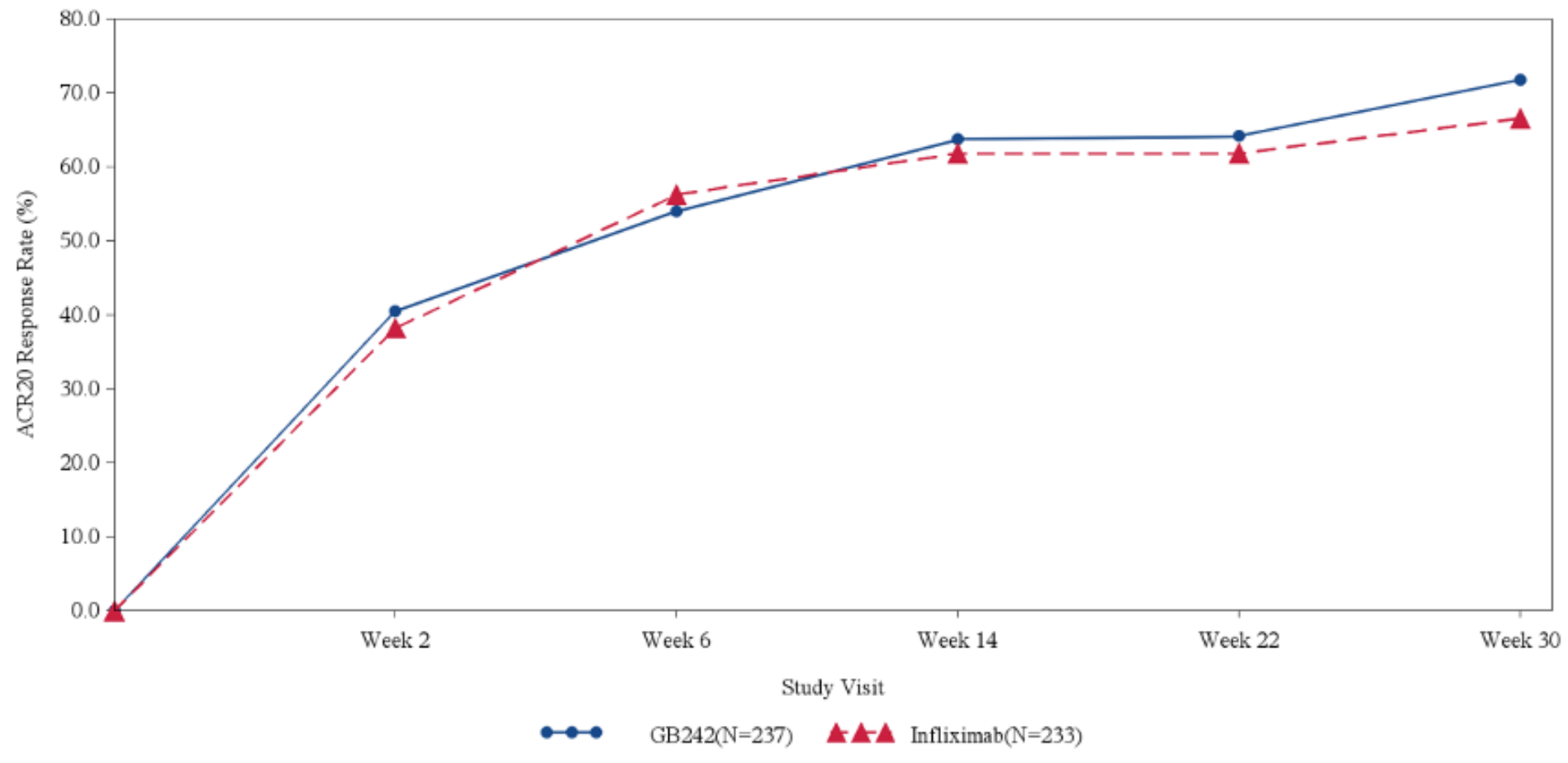

Figure 2

ACR20 response pattern over time. Note: INF: infliximab reference product 
A

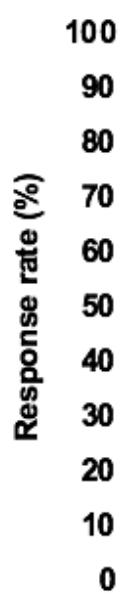

FAS

Treatment difference $=5.65 \%$

(95\%Cl: [ $-2.48 \%, 13.74 \%]$ )

$$
62.54
$$

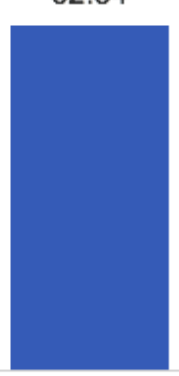

56.89

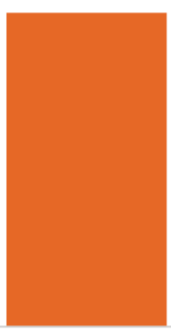

ACR20

-GB242 - INF

Treatment difference $=4.24 \%$

(95\% Cl: $[-3.71 \%, 12.17 \%])$

ACR50

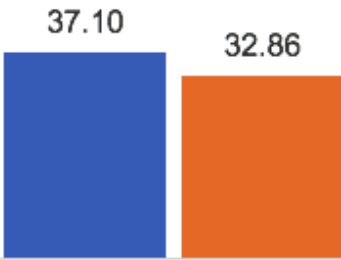

Treatment difference $=2.83 \%$ (95\%Cl: [-3.64\%, 9.32\%])

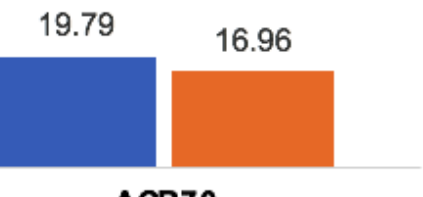

ACR70
B

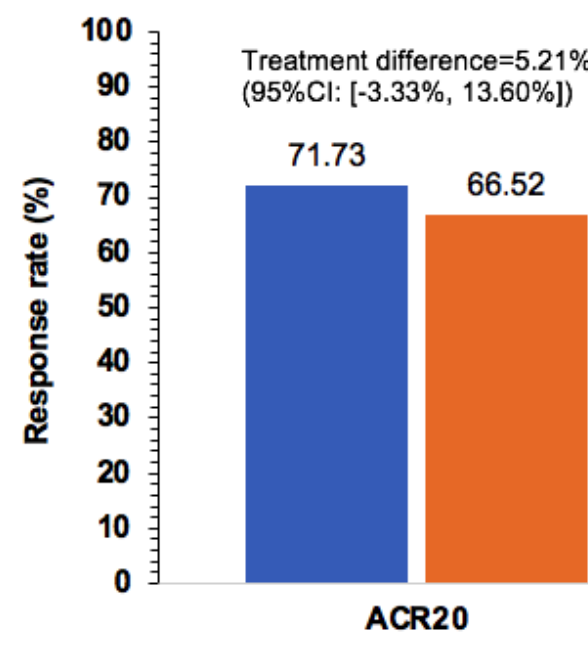

\section{PPS}
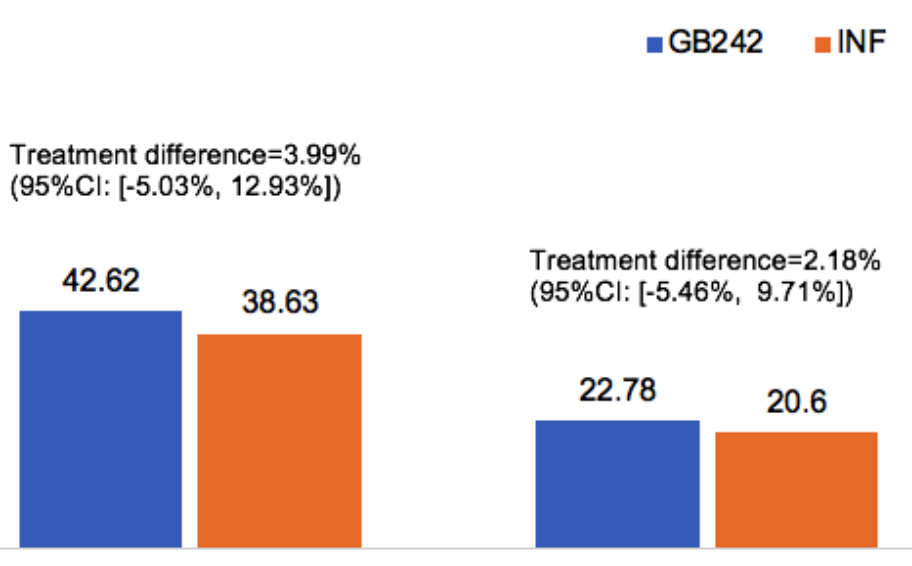

ACR50
Treatment difference $=\mathbf{2 . 1 8} \%$ (95\% Cl: [-5.46\%, 9.71\%])

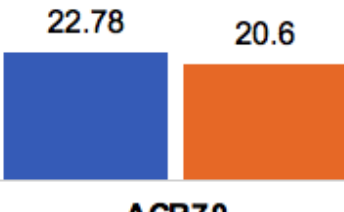

\section{Figure 3}

American College of Rheumatology (ACR) response rates at week 30. (A) ACR20, 50 and 70 responses for GB242 and INF in the full analysis set (FAS). (B) ACR20, 50 and 70 responses for GB242 and infliximab reference product (INF) in the per-protocol set (PPS). 

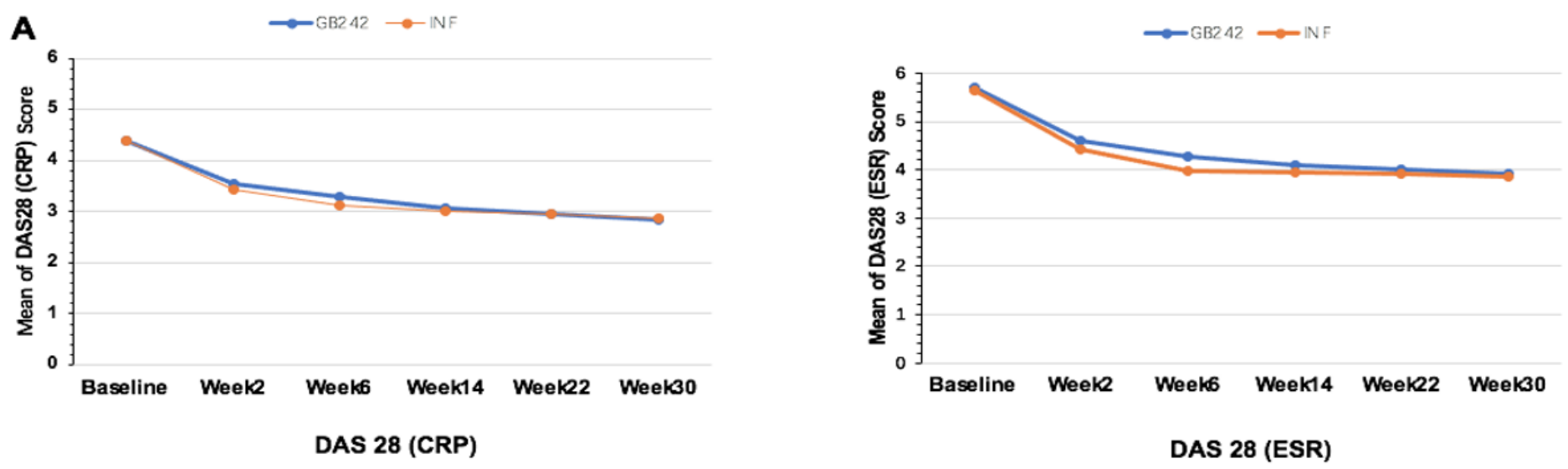

B DAS 28 (CRP)

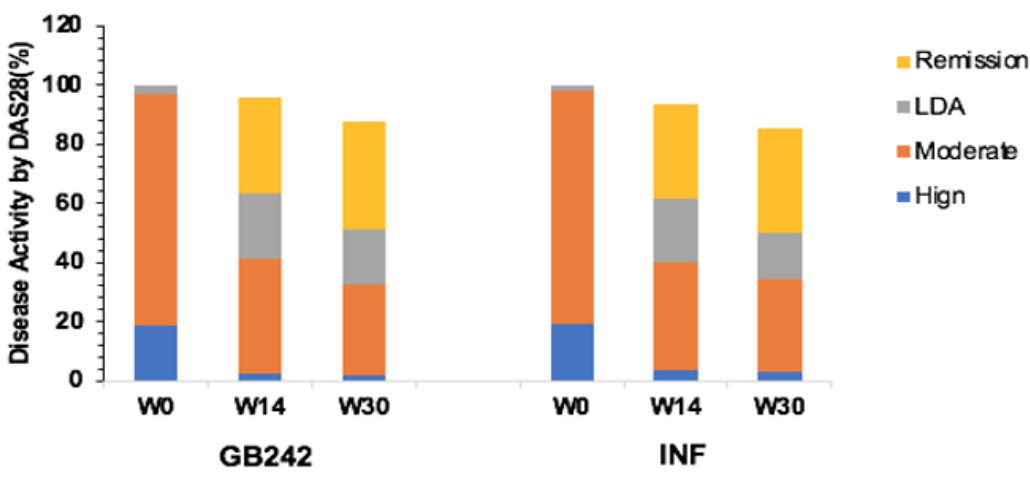

\section{Figure 4}

DAS28 responses in the per-protocol population. (A) Mean DAS28 score based on C reactive protein (CRP) and erythrocyte sedimentation rate (ESR) by baseline, weeks 14 and 30 for GB242 and infliximab reference product (INF). (B) Disease activity classification by DAS28. Remission is defined as DAS28<2.6, LDA is defined as DAS28 $2.6 \leq$ to $<3.2$, Moderate is defined as DAS28 $3.2 \leq$ to $\leq 5.1$ and high is defined as DAS28 $>5.1$.
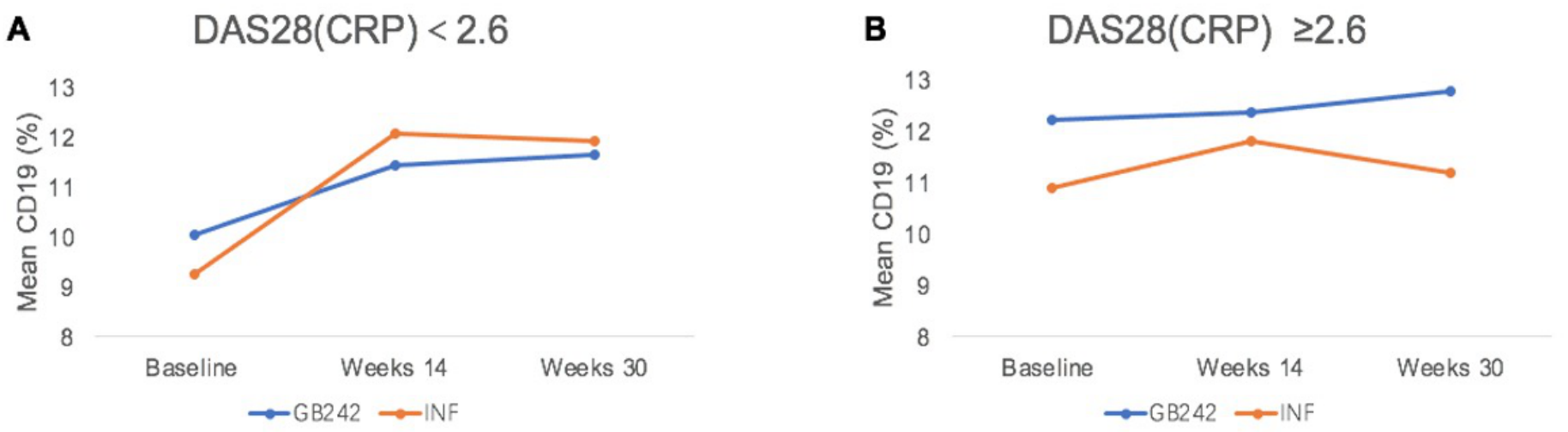

\section{Figure 5}

CD19 response pattern overtime. (A) Mean CD19 percentage based on DAS28<2.6 at weeks 30 for GB242 and infliximab reference product (INF). (B) Mean CD19 percentage based on DAS28 $\geq 2.6$ at weeks 30 for GB242 and 
IFN.

\section{Supplementary Files}

This is a list of supplementary files associated with this preprint. Click to download.

- GB242protocol.doc

- supplementaryfile.docx 